\title{
First-Principles Determination of Ultrahigh Thermal Conductivity of Boron Arsenide: A Competitor for Diamond?
}

\author{
L. Lindsay, ${ }^{1}$ D. A. Broido, ${ }^{2}$ and T. L. Reinecke ${ }^{1}$ \\ ${ }^{1}$ Naval Research Laboratory, Washington, D.C. 20375, USA \\ ${ }^{2}$ Department of Physics, Boston College, Chestnut Hill, Massachusetts 02467, USA
}

(Received 17 March 2013; published 8 July 2013)

\begin{abstract}
We have calculated the thermal conductivities $(\kappa)$ of cubic III-V boron compounds using a predictive first principles approach. Boron arsenide is found to have a remarkable room temperature $\kappa$ over $2000 \mathrm{~W} \mathrm{~m}^{-1} \mathrm{~K}^{-1}$; this is comparable to those in diamond and graphite, which are the highest bulk values known. We trace this behavior in boron arsenide to an interplay of certain basic vibrational properties that lie outside of the conventional guidelines in searching for high $\kappa$ materials, and to relatively weak phononisotope scattering. We also find that cubic boron nitride and boron antimonide will have high $\kappa$ with isotopic purification. This work provides new insight into the nature of thermal transport at a quantitative level and predicts a new ultrahigh $\kappa$ material of potential interest for passive cooling applications.
\end{abstract}

As microelectronic devices become smaller, faster, and more powerful, thermal management is becoming a critical challenge in, e.g., microprocessors, LEDs, and high power RF devices. As a result, the ability to identify and understand materials with high thermal conductivities $(\kappa)$ is becoming increasingly important [1]. Carbon based materials, including diamond and graphite, have long been recognized as having the highest $\kappa$ of any bulk material with room temperature (RT) values around $2000 \mathrm{~W} \mathrm{~m}^{-1} \mathrm{~K}^{-1}$ $[2,3]$. Other high $\kappa$ materials, such as copper, are not even close, having four to five times smaller values [4]. However, diamond is scarce and its synthetic fabrication suffers from slow growth rates, high cost, and low quality [5]. Thus it is of current interest to identify new materials with ultrahigh thermal conductivities.

Commonly accepted criteria [4] to guide the search for high $\kappa$ nonmetallic crystals include (i) simple crystal structure, (ii) low average atomic mass, $M_{\mathrm{av}}$, (iii) strong interatomic bonding, and (iv) low anharmonicity. Items (ii) and (iii) imply a large Debye temperature, $\theta_{D}$, and give the frequently used rule of thumb that $\kappa$ decreases with increasing $M_{\mathrm{av}}$ and with decreasing $\theta_{D}$. However, little progress has been made over the years in identifying new high $\kappa$ materials.

Until recently fully microscopic, parameter-free computational materials techniques for electronic properties have been more advanced than are those for thermal transport. In the last few years quantitative ab initio techniques have been developed for thermal transport [6-12], and we have made contributions to this development $[6,7,12]$. These techniques open the way to a fuller understanding of the key physical features in thermal transport and to the ability to predict accurately the $\kappa$ of new materials. Here, we present quantitative, first principles calculations of $\kappa$ for the class of boron based cubic III-V compounds, boron nitride (BN), boron phosphide (BP), boron arsenide (BAs), and boron antimonide (BSb), referred to collectively as $\mathrm{B} X$ compounds. We find that BAs has an exceptionally high RT $\kappa$ above $2000 \mathrm{~W} \mathrm{~m}^{-1} \mathrm{~K}^{-1}$, which is comparable to that of the bulk carbon crystals. This result is surprising given that a prediction based on the commonly accepted criteria above [4] gives RT $\kappa$ for BAs of only around $200 \mathrm{~W} \mathrm{~m}^{-1} \mathrm{~K}^{-1}$, comparable to that of silicon.

We trace these results to fundamental vibrational properties, some of which are not typically connected to prescriptions for high $\kappa$ in materials. The large arsenic to boron mass ratio and the unusual, almost purely covalent bonding in BAs give a large frequency gap between its acoustic and optic phonons ( $a-o$ gap) and give a bunching of the acoustic phonon dispersions; the intrinsic $\kappa$ is sensitive to the combination of these properties. In addition, the isotopically pure As atom gives relatively weak phononisotope scattering.

Thermal conductivity.-In semiconductors and insulators heat is carried primarily by the atomic vibrations of the lattice given by phonons [13]. The lattice thermal conductivity is a scalar for these cubic crystals and is given by

$$
\kappa=\frac{1}{V} \sum_{\lambda} C_{\lambda} v_{\lambda \alpha}^{2} \tau_{\lambda \alpha}
$$

where $V$ is the crystal volume, $C_{\lambda}=k_{B} n_{\lambda}^{0}\left(n_{\lambda}^{0}+1\right) \times$ $\left(\hbar \omega_{\lambda} / k_{B} T\right)^{2}$ is the specific heat per phonon mode, $k_{B}$ is the Boltzmann constant, and $\left.n_{\lambda}^{0}=1 /\left[\exp \left(\hbar \omega_{\lambda} / k_{B} T\right)-1\right)\right]$ is the Bose factor. The sum is over all phonon modes, $\lambda=(\mathbf{q}, j)$, with wave vector $\mathbf{q}$ and polarization $j$. Here, $\omega_{\lambda}$ is the phonon frequency. We define $\kappa_{\alpha \alpha}=\kappa$, and also $v_{\lambda \alpha}$ and $\tau_{\lambda \alpha}$ are the velocity component and the phonon transport lifetime along a cubic direction $\alpha$ for the assumed small $T$ gradients [14].

In our first principles approach, the phonon frequencies and velocities are determined by diagonalizing the dynamical matrix, and the phonon transport lifetimes are 
calculated using an iterative solution of the linearized Boltzmann transport equation for phonons [15]. The only inputs are the harmonic and anharmonic interatomic force constants, which are obtained using density functional theory $[16,17]$ and density functional perturbation theory [18]. The details have been published elsewhere $[6,7,12,19]$. In previous work we demonstrated good agreement with measured values of $\kappa$ for $\mathrm{Si}, \mathrm{Ge}$, diamond [6,7], $\mathrm{GaN}$ [12] and other III-V compound semiconductors [19] using no adjustable parameters.

The intrinsic lattice thermal conductivity, $\kappa_{\text {pure }}$, is governed by three-phonon scattering from the anharmonicity of the interatomic potential [13], and it is the upper bound to $\kappa$ for a non-metallic crystal. All scatterings must satisfy momentum and energy conservation, $\mathbf{q} \pm \mathbf{q}^{\prime}=\mathbf{q}^{\prime \prime}+\mathbf{K}$ and $\omega_{\lambda} \pm \omega_{\lambda^{\prime}}=\omega_{\lambda^{\prime \prime}}$, where $\mathbf{K}$ is a reciprocal lattice vector, which is zero for Normal processes and non-zero for resistive Umklapp processes. In high quality bulk materials around RT, the dominant thermal resistance for acoustic $(a)$ and optic $(o)$ phonons arises from this threephonon scattering, with the most important scattering channels involving three acoustic phonons ( $a a a)$ and two acoustic phonons combining with one optic phonon (aao) [20]. Scattering of phonons by isotopes can also play an important role in reducing $\kappa$ in some materials and is included using a standard perturbation theory approach [21,22] to determine $\kappa$ for naturally occurring isotope concentrations, $\kappa_{\text {nat }}$. Expressions for the intrinsic phonon-phonon scattering rates and phonon-isotope scattering rates have been given previously $[7,12,21]$. In real materials, sample-specific thermal resistance from extrinsic defects, grain boundaries, and other impurities can lower $\kappa$ from the values given here.

Our main results are given in Fig. 1 and Table I where the calculated $\kappa_{\text {nat }}$ for BAs is compared to those of BN, BP, $\mathrm{BSb}$, and diamond. Measured $\kappa$ for diamond and BP and other high $\kappa$ materials are included in Fig. 1, and measured $\mathrm{RT} \kappa$ values for diamond, $\mathrm{BP}, \mathrm{BN}, \mathrm{Si}$, and $\mathrm{Ge}$ are given in Table I. All the measured $\kappa$ values correspond to materials with naturally occurring isotope concentrations [27]. We note that no measurements of $\kappa$ for BAs and BSb have been reported to date; however, crystals of cubic BAs have been grown [28-30]. $\kappa_{\text {nat }}$ for BN, BP and BAs are higher than those of other non-carbon based high $\kappa$ materials. $\kappa_{\text {nat }}$ for BAs is seen to be considerably higher than those for all other materials except diamond, attaining an exceptionally high RT value of $2240 \mathrm{~W} \mathrm{~m}^{-1} \mathrm{~K}^{-1}$, over ten times higher than that predicted by conventional reasoning [4]. In addition, above RT it is larger than that for diamond. This is surprising given the criteria above because $M_{\mathrm{av}}$ of BAs is over three times larger than that of diamond and the calculated $\theta_{D}$ of BAs is about three times smaller (Table I).

Important hints to the underlying physics governing the high $\kappa$ of BAs are seen already in Fig. 1 and Table I: (i) the $\kappa$ for BAs and BSb drop less rapidly with temperature than

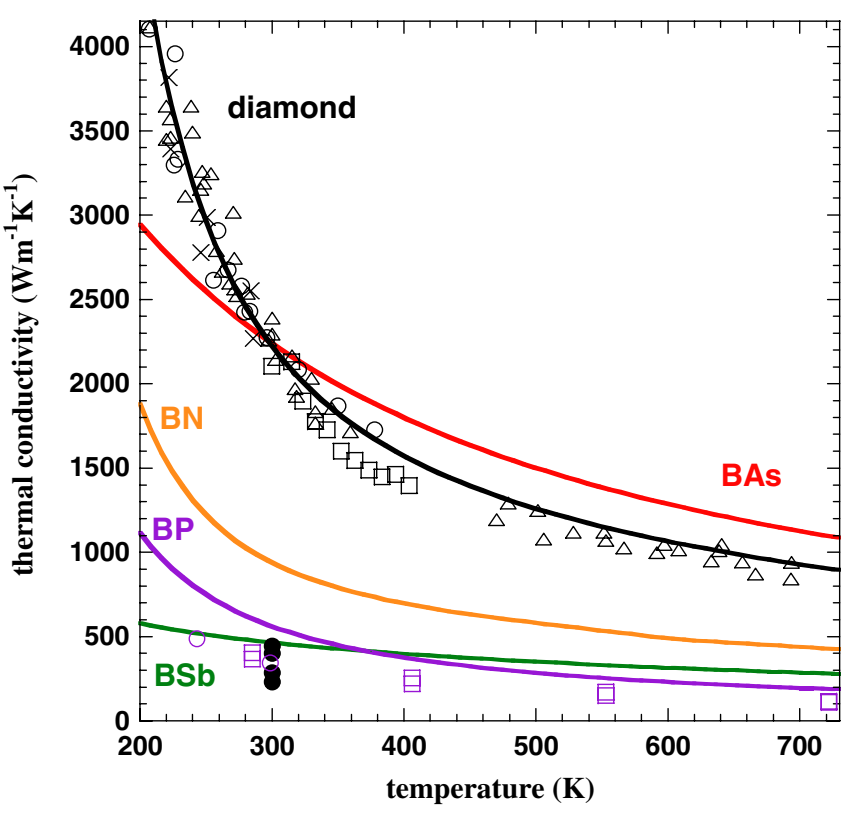

FIG. 1 (color online). Calculated $\kappa_{\text {nat }}$ vs temperature for cubic BN (orange), BP (purple), BAs (red), BSb (green), and diamond (black). Measured $\kappa$ values for diamond (open black squares [38], triangles [39], circles [2], and crosses [40]) and BP (open purple circles [4] and squares [24]) are given. Also shown are RT $(T=300 \mathrm{~K}) \kappa$ values (solid black circles) for common high $\kappa$ materials [41]: gallium nitride $\left(230 \mathrm{~W} \mathrm{~m}^{-1} \mathrm{~K}^{-1}\right)$, aluminum $\left(240 \mathrm{~W} \mathrm{~m}^{-1} \mathrm{~K}^{-1}\right)$, aluminum nitride $\left(285 \mathrm{~W} \mathrm{~m}^{-1} \mathrm{~K}^{-1}\right)$, copper $\left(400 \mathrm{~W} \mathrm{~m}^{-1} \mathrm{~K}^{-1}\right)$, and silicon carbide $\left(490 \mathrm{~W} \mathrm{~m}^{-1} \mathrm{~K}^{-1}\right)$.

they do for diamond, BN and BP; (ii) as the anion changes from $\mathrm{N}$ to $\mathrm{P}$ to $\mathrm{As}$ and to $\mathrm{Sb}, M_{\mathrm{av}}$ increases and $\theta_{D}$ decreases monotonically, but the RT $\kappa$ fluctuates. For the group IV materials, $\kappa$ decreases monotonically with

TABLE I. $M_{\mathrm{av}}$ and calculated $\theta_{D}$, RT $\kappa_{\text {nat }}$, and $\kappa_{\text {pure }}$, and percent enhancement to $\kappa$ with isotopic purification given by $P=100 \times\left(\kappa_{\text {pure }} / \kappa_{\text {nat }}-1\right)$. The naturally occurring isotope concentrations used to determine $\kappa_{\text {nat }}$ for these materials were $\left(19.9 \%{ }^{10} \mathrm{~B}, 80.1 \%{ }^{11} \mathrm{~B}\right),\left(98.1 \%{ }^{12} \mathrm{C}, 1.1 \%{ }^{13} \mathrm{C}\right),\left(57.2 \%{ }^{121} \mathrm{Sb}\right.$, $\left.42.8 \%{ }^{123} \mathrm{Sb}\right),\left(92.2 \%{ }^{28} \mathrm{Si}, 4.7 \%{ }^{29} \mathrm{Si}, 3.1 \%{ }^{30} \mathrm{Si}\right)$, and $(20.4 \%$ $\left.{ }^{70} \mathrm{Ge}, 27.3 \%{ }^{72} \mathrm{Ge}, 7.8 \%{ }^{73} \mathrm{Ge}, 36.7 \%{ }^{74} \mathrm{Ge}, 7.8 \%{ }^{76} \mathrm{Ge}\right)$. All other elements are isotopically pure. Measured values for $c$-BN [23], BP [24], and high quality diamond [2], Si [25], and Ge [26] with naturally occurring isotope concentrations are given in parentheses in the $\kappa_{\text {nat }}$ column.

\begin{tabular}{lcrccr}
\hline \hline & $\begin{array}{l}M_{\text {av }} \\
(\mathrm{amu})\end{array}$ & $\begin{array}{c}\theta_{D} \\
(\mathrm{~K})\end{array}$ & $\begin{array}{c}\kappa_{\text {nat }} \\
\left(\mathrm{W} \mathrm{m}^{-1} \mathrm{~K}^{-1}\right)\end{array}$ & $\begin{array}{c}\kappa_{\text {pure }} \\
\left(\mathrm{W} \mathrm{m}^{-1} \mathrm{~K}^{-1}\right)\end{array}$ & $P$ \\
\hline$c$-BN & 12.41 & 2025 & $940(768)$ & 2145 & 130 \\
$\mathrm{BP}$ & 20.89 & 1110 & $580(400)$ & 665 & 15 \\
$\mathrm{BAs}$ & 42.87 & 700 & 2240 & 3170 & 40 \\
BSb & 66.28 & 495 & 465 & 1180 & 155 \\
Diamond & 12.01 & 2280 & $2290(2270)$ & 3450 & 50 \\
$\mathrm{Si}$ & 28.09 & 710 & $145(142)$ & 155 & 7 \\
$\mathrm{Ge}$ & 72.59 & 415 & $60(59)$ & 75 & 23 \\
\hline \hline
\end{tabular}


increasing $M_{\mathrm{av}}$ following the expected behavior. This is associated with the heavier mass and reduced $\theta_{D}$ that result in decreased acoustic phonon velocities, decreased frequencies, and in increased phonon-phonon scattering by increasing phonon populations in going from diamond to $\mathrm{Si}$ and to Ge. The $\mathrm{B} X$ compounds initially follow a similar trend with $\kappa$ dropping from BN to BP (Table I). However, this is followed by a sharp rise to BAs before dropping to $\mathrm{BSb}$. This unusual behavior of $\kappa$ for the $\mathrm{B} X$ materials is not reflected in their harmonic and anharmonic IFCs, which have monotonically decreasing magnitudes with increasing $M_{\mathrm{av}}$, similar to those of the group IV materials (see Table A of the Supplemental Material [22]).

We find that the unusual behavior of the intrinsic $\kappa$ arises largely from the interplay of certain basic properties, including (i) a large frequency gap between acoustic and optic phonons ( $a-O$ gap) and (ii) the bunching of the acoustic branches, which are not in the simple criteria mentioned above [31].

a-o gap.- - In going from $\mathrm{N}$ to $\mathrm{P}$ to $\mathrm{As}$ to $\mathrm{Sb}$ the increasing $M_{X} / M_{B}$ ratio opens successively larger $a$ - $o$ gaps in the BX compounds (see Fig. 2 for BAs). This is familiar from the diatomic linear chain model where the scale of the acoustic (optic) phonon branch is governed by the larger (smaller) mass. While heat is carried primarily by the lower frequency acoustic phonons, the optic phonons provide important scattering channels for the acoustic modes

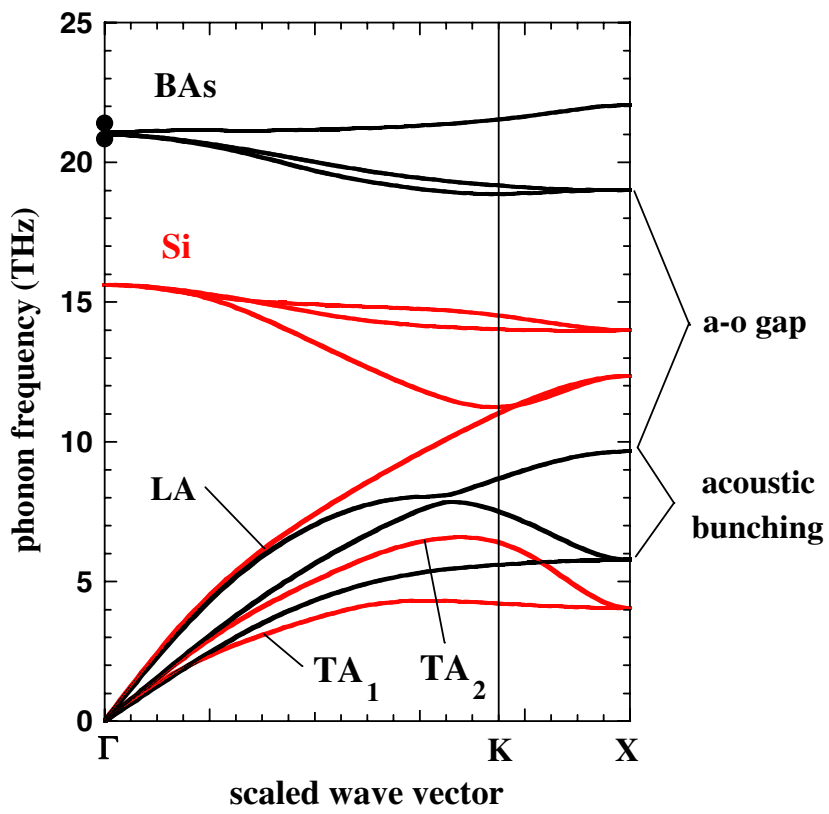

FIG. 2 (color online). Phonon frequency vs scaled wave vector in the high symmetry $\Gamma \rightarrow \mathrm{K} \rightarrow \mathrm{X}$ direction for BAs (black) and Si (red) with experimental Raman data for BAs (black circles) [29]. BAs has a large $a-o$ frequency gap, which is absent in Si. Note that the near-zone center acoustic velocities for BAs and $\mathrm{Si}$ are similar. However, the three acoustic branches [two transverse $\left(\mathrm{TA}_{1}\right.$ and $\mathrm{TA}_{2}$ ) and one longitudinal (LA)] are bunched closer to one another in BAs than are the corresponding branches in Si. in many materials, particularly through aao scattering from which a large fraction of resistive Umklapp scattering derives. For aao scattering, the sum of the two acoustic phonon frequencies must equal the optic phonon frequency. In BAs and $\mathrm{BSb}$, aao scattering is prohibited by the conservation conditions, which reduces their intrinsic thermal resistances.

The absence of aao scattering affects the $T$ dependence of $\kappa$ for BAs and BSb as seen in Fig. 1. At low T, aao scattering is frozen out in all materials. With increasing temperature, the more rapid decrease in $\kappa(T)$ for diamond, $\mathrm{BN}$, and $\mathrm{BP}$ is a signature that the aao scattering is increasing as optic phonons are thermally populated. In contrast, aao scattering is absent at all temperatures in $\mathrm{BAs}$ and $\mathrm{BSb}$, thus giving weaker temperature dependence on $\kappa$.

Bunching of the acoustic branches. - Figure 2 shows that in their higher frequency range ( $4 \mathrm{THz}$ to $10 \mathrm{THz}$ ) the three acoustic branches are closer to one another in BAs than they are in Si. The bunching of the acoustic branches is present in all of the BX compounds, consistent with previous calculations [32], and is also present in diamond. It is connected to the unusual nature of the bonding in these systems $[33,34]$. This bunching decreases the phase space for the higher frequency aa $a$ scattering processes, particularly for resistive Umklapp processes because they tend to involve higher frequency phonons. This is similar to a simple argument that gives that three phonons from the same dispersive acoustic branch cannot simultaneously satisfy momentum and energy conservation $[13,35]$. Thus in the limit that the acoustic branches coincide, no $a a a$ scattering is possible. In addition to the reduced phase space, the scattering strengths of many of the remaining processes are considerably weakened by the acoustic bunching. To see this, note that since the bunched acoustic branches are similar in energy, an $a a a$ scattering initiated in the higher-frequency range involves two phonons of similar high frequency, $\omega_{\lambda}$ and $\omega_{\lambda^{\prime \prime}}$, and one phonon of small frequency, $\omega_{\lambda^{\prime}}$, and thus small wave vector magnitude, $q^{\prime}$. In this case the scattering matrix elements scale as $\left|\Phi_{\lambda \lambda^{\prime} \lambda^{\prime \prime}}\right|^{2} \sim q^{\prime 2}$. As the branches bunch together, $q^{\prime}$ and the matrix elements become smaller and the resulting $a a a$ scattering becomes weaker.

BAs is unusual in that the acoustic branch bunching weakens $a a a$ scattering, its $a-o$ gap is large enough to freeze out all aao processes, and its stiff bonding and small $M_{B}$ give a relatively large $\theta_{D}$. This combination of features is not present in the other materials and gives BAs anomalously large intrinsic phonon transport lifetimes and correspondingly large contributions to $\kappa$ from the mid-high frequency acoustic phonons which are ultimately responsible for the ultrahigh $\kappa$. This behavior is seen by the large peak in Fig. 3, which plots the calculated contribution to $\kappa_{\text {pure }}$ as a function of frequency, $\kappa(\nu)$, for BAs and Si [36]. While the frequency scale of the Si acoustic spectrum 


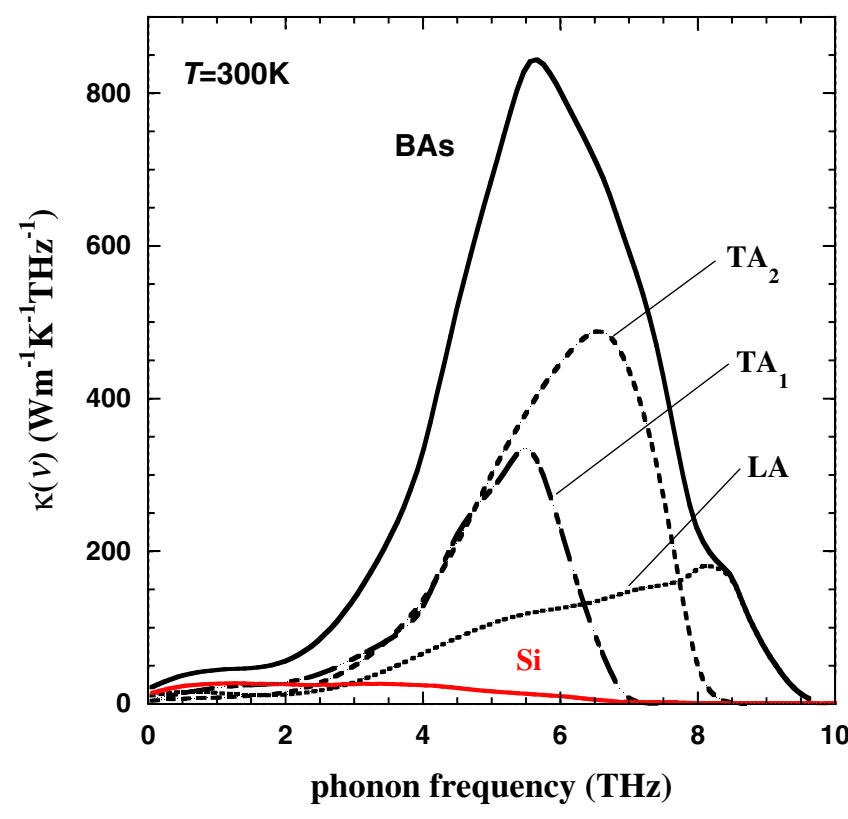

FIG. 3 (color online). Calculated contributions to the intrinsic thermal conductivity $\kappa(\nu)$ vs $\nu$ for Si (solid red curve) and BAs (solid black curve) [36]. The per branch contributions to $\kappa(\nu)$ for the $\mathrm{TA}_{1}$ (dashed-dotted black curve), the $\mathrm{TA}_{2}$ (dashed black curve), and LA (dotted black curve) phonon branches are also shown for BAs. The areas under the solid curves give the RT $\kappa_{\text {pure }}$ for each material. In BAs, the $\mathrm{TA}_{2}$ branch gives the greatest contribution because its phonon modes in the middle of the high frequency range have high group velocities where the $\mathrm{TA}_{2}$ phonon transport lifetimes are large.

shown in Fig. 2 is somewhat larger than that for BAs, Si has no $a-o$ gap, and its acoustic branches are well separated compared to those in BAs. This gives strong $a a a$ and $a a o$ scattering in Si. As a result, contributions to the Si $\kappa_{\text {pure }}$ come from a lower frequency range and are much smaller than for BAs as seen in Fig. 3.

Isotopic scattering can play an important role in determining $\kappa$ in many material systems, such as those considered here. BAs has a large mixture of B isotopes, however, it has relatively small contributions from isotopic scattering, smaller even than cubic BN, BSb, and diamond (Table I). The reason for this is that for BAs, the large As to $\mathrm{B}$ mass ratio causes the atomic motion of the short wavelength acoustic phonons, which give the largest contributions to $\kappa$, to be dominated by the heavier As mass [21,35], and As happens to be isotopically pure. As a result, both the RT $\kappa_{\text {pure }}$ and $\kappa_{\text {nat }}$ for BAs are similar to that of diamond (Table I).

It is interesting to compare the $\kappa_{\text {pure }}$ and $\kappa_{\text {nat }}$ of BAs to those of $\mathrm{BN}$ and $\mathrm{BSb}$ (Table I). Cubic BN is a conventional high $\kappa$ material. Its light constituent atoms and stiff covalent bonding give it a large RT $\kappa_{\text {pure }}$ of about $2100 \mathrm{~W} \mathrm{~m}^{-1} \mathrm{~K}^{-1}$. In contrast, the $\kappa_{\text {pure }}$ of about $1200 \mathrm{~W} \mathrm{~m}^{-1} \mathrm{~K}^{-1}$ for $\mathrm{BSb}$ arises from the lack of aao scattering and weak $a a a$ scattering, and it would not be predicted from conventional reasoning, similar to BAs. However, BSb has a larger anion mass, a smaller $\theta_{D}$, and smaller acoustic velocities, which are responsible for its $\kappa_{\text {pure }}$ being less than half that of BAs. Inclusion of natural isotope concentrations has a stronger effect in $\mathrm{BN}$ and $\mathrm{BSb}$ compared to that of BAs. For BN, the B and $\mathrm{N}$ masses are similar while for BSb the heavy $\mathrm{Sb}$ atoms have a large isotope mix. In both cases the motion of atoms with large isotope mixtures (B in $\mathrm{BN}$ and $\mathrm{Sb}$ in $\mathrm{BSb}$ ) for the short wavelength acoustic phonons causes stronger isotope scattering and gives these materials much larger percentage reductions in $\kappa$ compared to BAs.

In summary, we have calculated the thermal conductivities of cubic III-V boron based crystals using an $a b$ initio Boltzmann transport approach. We find that BAs has an exceptionally high $\kappa\left(\kappa_{\text {nat }}>2000 \mathrm{~W} \mathrm{~m}^{-1} \mathrm{~K}^{-1}, \kappa_{\text {pure }}>\right.$ $3000 \mathrm{~W} \mathrm{~m}^{-1} \mathrm{~K}^{-1}$ at $\mathrm{RT}$ ) comparable to that of diamond, which has the highest bulk RT $\kappa$ of any known material. This surprising result stems in part from the combination of a bunching of the acoustic phonon dispersions, a large frequency gap between acoustic and optic phonon branches and relatively weak phonon-isotope scattering. Isotopically pure $\mathrm{BN}$ and $\mathrm{BSb}$ are also found to have very high $\kappa$ with RT values around $2000 \mathrm{~W} \mathrm{~m}^{-1} \mathrm{~K}^{-1}$ and $1200 \mathrm{~W} \mathrm{~m}^{-1} \mathrm{~K}^{-1}$, respectively. Efficient isotope enrichment technology could make $\mathrm{BN}$ and $\mathrm{BSb}$ also viable high $\kappa$ materials.

This work illustrates the power of quantitative computational techniques for thermal transport and identifies BAs as an ultrahigh $\kappa$ material that may open new opportunities for passive cooling applications [37]. It gives important new insight into the physics of thermal conductivity in solids, which could provide useful guidance in the design of future high $\kappa$ materials.

This work was supported in part by ONR and DARPA (L.L. and T.L.R.). L. L. acknowledges support from the NRC-NRL Research Associateship Program. D. A. B acknowledges support primarily from the S3TEC, an Energy Frontier Research Center funded by the U.S. Department of Energy, Office of Science, Office of Basic Energy Sciences under Award No. DE-FG02-09ER46577, and also from the National Science Foundation under Grant No. 1066634.

[1] P. Ball, Nature (London) 492, 174 (2012).

[2] L. Wei, P. K. Kuo, R. L. Thomas, T. R. Anthony, and W. F. Banholzer, Phys. Rev. Lett. 70, 3764 (1993).

[3] G. A. Slack, Phys. Rev. 127, 694 (1962).

[4] G. A. Slack, J. Phys. Chem. Solids 34, 321 (1973).

[5] Graphite has weak interlayer van der Waals bonding which makes $\kappa$ highly anisotropic and the material mechanically soft, and thus is not appropriate for many applications.

[6] D. A. Broido, M. Malorny, G. Birner, N. Mingo, and D. A. Stewart, Appl. Phys. Lett. 91, 231922 (2007). 
[7] A. Ward, D. A. Broido, D. A. Stewart, and G. Deinzer, Phys. Rev. B 80, 125203 (2009).

[8] X. Tang and J. Dong, Proc. Natl. Acad. Sci. U.S.A. 107, 4539 (2010).

[9] J. Garg, N. Bonini, B. Kozinsky, and N. Marzari, Phys. Rev. Lett. 106, 045901 (2011).

[10] J. Shiomi, K. Esfarjani, and G. Chen, Phys. Rev. B 84, 104302 (2011).

[11] Z. Tian, J. Garg, K. Esfarjani, T. Shiga, J. Shiomi, and G. Chen, Phys. Rev. B 85, 184303 (2012).

[12] L. Lindsay, D. A. Broido, and T. L. Reinecke, Phys. Rev. Lett. 109, 095901 (2012).

[13] J. M. Ziman, Electrons and Phonons (Oxford University, London, 1960).

[14] The phonon transport lifetime is defined from the calculated nonequilibrium distribution function, as described in Refs. [6,7,15].

[15] M. Omini and A. Sparavigna, Nuovo Cimento Soc. Ital. Fis. 19D, 1537 (1997).

[16] P. Hohenberg and W. Kohn, Phys. Rev. 136, B864 (1964).

[17] W. Kohn and L. J. Sham, Phys. Rev. 140, A1133 (1965).

[18] S. Baroni, S. Gironcoli, A. D. Corso, and P. Giannozzi, Rev. Mod. Phys. 73, 515 (2001).

[19] L. Lindsay, D. A. Broido, and T. L. Reinecke, Phys. Rev. B 87, 165201 (2013).

[20] ooo processes are forbidden in most materials because three optic phonons usually cannot satisfy energy conservation. aoo scattering, while present, is severely restricted in most materials by the combined conservation conditions.

[21] S. I. Tamura, Phys. Rev. B 30, 849 (1984).

[22] See Supplemental Material at http://link.aps.org/ supplemental/10.1103/PhysRevLett.111.025901 for discussion of the coefficient of thermal expansion, the isotope scattering rates, and calculated harmonic and anharmonic IFCs.

[23] N. V. Novikov, T. D. Osetinskaya, A. A. Shul'zhenko, A. P. Podoba, A. N. Sokolov, and I. A. Petrusha, Dopov. Akad. Nauk Ukr. RSR, Ser. A: Fiz.-Tekh. Mat. Nauki 72 (1983).

[24] Y. Kumashiro, T. Mitsuhashi, S. Okaya, F. Muta, T. Koshiro, Y. Takahashi, and M. Mirabayashi, J. Appl. Phys. 65, 2147 (1989).

[25] A. V. Inyushkin, A. N. Taldenkov, A. M. Gibin, A. V. Gusev, and H.-J. Pohl, Phys. Status Solidi C 1, 2995 (2004).

[26] V. I. Ozhogin, A. V. Inyushkin, A. N. Taldenkov, A. V. Tikhomirov, G. E. Popov, E. Haller, and K. Itoh, JETP Lett. 63, 490 (1996).

[27] Calculated $\kappa_{\text {nat }}$ values are in good agreement with measured $\kappa$ values for high quality $\mathrm{Si}, \mathrm{Ge}$, and diamond samples, as was found previously in Refs. [6,7]. For BN, measured RT $\kappa$ values range from $180 \mathrm{~W} \mathrm{~m}^{-1} \mathrm{~K}^{-1}$ to $768 \mathrm{~W} \mathrm{~m}^{-1} \mathrm{~K}^{-1}$, likely reflecting a wide range of sample quality and crystallite size. Our calculated RT $\kappa_{\text {nat }}$ for BN is about $20 \%$ higher than the highest measured RT $\kappa$ value (Table I). For BP, our calculated RT $\kappa_{\text {nat }}$ is $45 \%$ higher than the corresponding measured values. Slack [4] previously noted that little is known about the impurities in the BP samples. Since impurities (other than isotopic impurities) and other defects reduce $\kappa$ and are not included in our theory, the calculated $\kappa$ values for $\mathrm{BN}$ and $\mathrm{BP}$ are expected to lie above the measured values.

[28] S. M. Ku, J. Electrochem. Soc. 113, 813 (1966).

[29] R. G. Greene, H. Luo, A. L. Ruoff, S. S. Trail, and F. I. DiSalvo, Phys. Rev. Lett. 73, 2476 (1994).

[30] S. Wang, S. F. Swingle, H. Ye, F.-R. F. Fan, A. H. Cowley, and A. J. Bard, J. Am. Chem. Soc. 134, 11056 (2012).

[31] Also contributing to the high- $\kappa$ values are the light boron mass and stiff covalent bonding (almost pure in BAs), which give high overall frequency scales and large acoustic velocities in the $\mathrm{B} X$ materials compared to other III-V compounds.

[32] T. Pletl, Ph.D. thesis, University of Regensburg, 1998; S. Bağci, S. Duman, H. M. Tütüncü, and G. P. Srivastava, Phys. Rev. B 79, 125326 (2009).

[33] P. Pavone, K. Karch, O. Schutt, W. Windl, D. Strauch, P. Giannozzi, and S. Baroni, Phys. Rev. B 48, 3156 (1993).

[34] A. Fukumoto, Phys. Rev. B 42, 7462 (1990).

[35] M. Lax, P. Hu, and V. Narayanamurti, Phys. Rev. B 23, 3095 (1981).

[36] $\kappa=\int \kappa(\nu) d \nu$ where $\kappa(\nu)=1 / V \sum_{\lambda} C_{\lambda} v_{\lambda \alpha}^{2} \tau_{\lambda \alpha} \delta\left(\nu-\nu_{\lambda}\right)$. $\kappa(\nu) d \nu$ gives the contribution to the calculated $\kappa$ in the frequency range between $\nu$ and $\nu+d \nu$.

[37] BAs has a desirable coefficient of thermal expansion (CTE), close to that of Si, the most widely used material in the semiconductor industry. This is essential for passive cooling of electronics to avoid introducing thermal stresses [22].

[38] D. G. Onn, A. Witek, Y.Z. Qiu, T. R. Anthony, and W.F. Banholzer, Phys. Rev. Lett. 68, 2806 (1992).

[39] J. R. Olson, R. O. Pohl, J. W. Vandersande, A. Zoltan, T. R. Anthony, and W.F. Banholzer, Phys. Rev. B 47, 14850 (1993).

[40] R. Berman, P. R. W. Hudson, and M. Martinez, J. Phys. C 8, L430 (1975).

[41] G. A. Slack, L. J. Schowalter, D. Morelli, and J. A. Freitas, J. Cryst. Growth 246, 287 (2002); G. A. Slack, R. A. Tanzilli, R. O. Pohl, and J. W. Vandersande, J. Phys. Chem. Solids 48, 641 (1987); G. A. Slack, J. Appl. Phys. 35, 3460 (1964). 